\title{
riccafd
}

Revista Iberoamericana de Ciencias de la Actividad Fisica y el Deporte

\section{MUERTE SÚBITA EN EL DEPORTE, PROPUESTA DE INTERVENCIÓN TEMPRANA}

\section{SUDDEN DEATH IN SPORT, PROPOSAL FOR EARLY INTERVENTION}

\author{
Erazo Martínez, Oscar Felipe ${ }^{1}$ y Alvarez Ríos, Jorge Norvey ${ }^{2}$
}

${ }^{1}$ Oscar Felipe Erazo Martínez. Médico; Estudiante Maestría en Enseñanza de las Ciencias; Docente Universidad de Caldas - Colombia; Oscar.erazo@ucaldas.edu.co

${ }^{2}$ Jorge Norvey Alvarez Ríos. Magister en Educación. Docencia; Estudiante Doctorado en Educación. Licenciado en Educación Física, Recreación y Deporte; Docente Universidad de Caldas - Colombia; Jorge.alvarez@ucaldas.edu.co

Código UNESCO: 3210. Medicina preventiva

Clasificación Consejo de Europa: 11. Medicina del Deporte

Recibido el 12 de diciembre de 2017

Aceptado el 25 de febrero de 2018

Correspondencia:

Jorge Norvey Alvarez Ríos

jorge.alvarez@ucaldas.edu.co

DOI: http://dx.doi.org/10.24310/riccafd.2018.v7i1.4857

\section{RESUMEN}

A pesar de las bajas tasas de ocurrencia, la muerte súbita se produce en deportistas sanos e incluso en eventos deportivos de alto rendimiento a nivel mundial. Su etiología se atribuye principalmente a enfermedades cardiovasculares y patologías congénitas. El objetivo principal de este artículo es realizar una recopilación y revisión de artículos especializados para establecer el estado del arte en esta materia. Una búsqueda especializada se realizó en las bases de datos PubMed, Science Direct, Scielo, Redalyc y La Referencia, identificando 43.608 artículos relacionados seleccionando 29 de ellos que cumplen ciertos criterios de selección adoptados. Finalmente, las principales causas, síntomas y factores desencadenantes de muerte súbita son identificados y analizados, y se describen los procedimientos orientados a la prevención y el manejo adecuado de estos eventos, así como el entrenamiento necesario de habilidades y destrezas dirigidos a los primeros respondientes.

Palabras claves: deporte, muerte, medicina del deporte (Tesauro de la UNESCO). 


\begin{abstract}
Despite the low rates of occurrence, Sudden Death occurs in healthy athletes and even in high performance sports events worldwide. Its etiology is attributed mainly to cardiovascular diseases and congenital pathologies. The main objective of this article, is to compile and review specialized articles to establish the state-of-the-art in this matter. A specialized search was conducted in PubMed, Science Direct, Scielo, Redalyc and La Referencia databases, identifying 43.608 related articles, selecting 29 of them which meet certain adopted selection criteria. Finally, the main causes, symptoms and trigger factors of Sudden Death are identified and analyzed, and the procedures oriented to prevention and proper management of these events, as well as the necessary training of capabilities and skills aimed at the first respondents, are described.
\end{abstract}

Key words: sport, death, sports medicine

\title{
INTRODUCCIÓN
}

El deporte y la actividad física se consolidan como una de las principales estrategias para disminuir los factores de riesgo, entre ellos, el cardiovascular. Pero de manera particular, este también, puede desencadenar la muerte durante su desarrollo o minutos después, en lo que se conoce como muerte súbita ${ }^{(1)}$. Estos eventos no solo ocurren en escenarios de alta competencia, por el contrario muchos de los casos suceden durante el entrenamiento. Por lo anterior, es fundamental desarrollar estrategias de prevención e identificación de los factores de riesgo.

La revisión de la evidencia científica más reciente, muestra que las enfermedades cardiovasculares y algunas patologías congénitas, favorecen el desarrollo de la muerte súbita. Es por esto, que es necesario la valoración médica y exámenes previos al inicio de un programa de entrenamiento, entre ellos el desarrollo de electrocardiografía, entre otros.

Finalmente, es necesario el entrenamiento de habilidades y destrezas en las maniobras de reanimación cardiopulmonar (RCP). Este entrenamiento dirigido a los entrenadores, los deportistas y en general en todo el equipo interdisciplinario que componen los clubes deportivos. Lo anterior, desde un perfil de primer respondiente, previo al arribo del sistema de emergencias.

A partir de estas reflexiones teóricas y consultas previas, se da inicio a esta revisión bibliográfica, con el fin de analizar y describir los principales aportes científicos para prevenir y atender de manera inicial la muerte súbita en el deportista. Esta búsqueda se realizó mediante el uso de bases de datos científicas, las cuales aportaron artículos determinantes para conocer la incidencia, las causas, la prevención y el manejo inicial de la paro cardiorrespiratorio en el deportista. 


\section{MATERIALES Y MÉTODOS}

Se utilizó la metodología de revisión de artículos científicos, los cuales fueron acotados en espacio, tiempo y lugar. Se utilizó la combinación de términos específicos de búsqueda, los cuales surgieron de los Descriptores de Ciencias de la Salud - DECs y Tesauro de la UNESCO (2).

Los criterios de inclusión fueron: artículos en inglés, español y portugués disponibles del año 2012 en adelante, estudios en personas, estudios internacionales, nacionales y locales. Las palabras claves empleadas fueron muerte súbita, resucitación cardiopulmonar y muerte súbita en el deporte; de igual forma en inglés, sudden death, sudden death in sport y cardiopulmonary resuscitation. Los resultados fueron:

Tabla 1. Número de artículos seleccionados

\begin{tabular}{lcc}
\hline Revista & $\begin{array}{c}\text { Resultados de } \\
\text { la búsqueda de } \\
\text { artículos }\end{array}$ & Seleccionados \\
\hline PUBMED & 484 & 9 \\
Science Direct & 1180 & 12 \\
Scielo & 200 & 3 \\
Redalyc & 41742 & 3 \\
La Referencia & 2 & 2 \\
\hline TOTAL ARTICULOS & & $\mathbf{2 9}$ \\
\hline
\end{tabular}

En total se utilizaron 29 artículos científicos identificados en las bases de datos, de los cuales surgen las categorías de discusión y análisis. Muchos de los artículos que no cumplieron con los criterios de inclusión, fueron aquellos que están documentados con otro énfasis diferente a los propuestos en la revisión.

\section{RESULTADOS Y DISCUSIÓN}

\section{PERFIL EPIDEMIOLÓGICO}

En la actualidad existe un aumento de personas que realizan actividad física; ya sea en el campo escolar, laboral o militar. Con esto, se incrementa la probabilidad de ocurrencia de lesiones e incluso la muerte súbita de los deportistas en escenarios deportivos ${ }^{(3)}$. La muerte súbita se consolida como un problema para las ciencias del deporte, representado en las tasas e índices de mortalidad. Estos eventos ocurren, en algunos casos, en personas sanas pero que tienen factores de riesgo coronario, entre ellos la hipercolesterolemia y la diabetes ${ }^{(4)}$.

La muerte súbita es definida como una muerte inesperada y repentina debido a causas naturales, en pacientes con un corazón estructuralmente normal. Algunos autores dentro de su definición solo incluyen las muertes en la primera hora después del inicio de los síntomas; otros, documentan casos de 
muerte en las 24 horas después de haber ocurrido el episodio y, existen adicionalmente, conceptos que describen esta situación en las primeras seis horas de inicio de los síntomas ${ }^{(5-9)}$.

La muerte súbita en el deporte, se considera como un evento inesperado por causas naturales que ocurre en una práctica deportiva e incluso después de las primeras 24 horas del inicio de los síntomas. Algunos autores, incluyen además en el concepto de muerte súbita, aquellos casos en los cuales existe un paro cardiorrespiratorio al cual se le ha brindado reanimación cardiopulmonar y se ha logrado la supervivencia ${ }^{(5-8)}$. El no contar con un consenso en la definición, ha generado dificultades para realizar seguimiento epidemiológico a la muerte súbita en el deporte.

En la actualidad, se calcula la incidencia de la muerte súbita en deportistas entre 1/1.000.000 y 1/23.000 atletas por año. La Asociación Americana del Corazón, calcula que prevalencia de un trastorno cardiovascular subyacente, que favorece la ocurrencia de la muerte súbita en jóvenes atletas es de $0,3 \%$ o 1 en 333. La causa más común de este evento en deportistas menores de 40 años, es la miocardiopatía hipertrófica. Además, las anomalías genéticas son otra de las causas en personas jóvenes, las cuales conforman el $3 \%$ de todas las muertes, es así como en el sudeste asiático el síndrome de Brugada conforma el $25 \%$ de las muertes en los deportistas (4,10-12). Las anteriores cifras varían de manera significativa, en la actualidad no existen datos consolidados que permita evidenciar la problemática de manera clara ${ }^{(5,7,13)}$.

\section{ETIOPATOLOGÍA DE LA MUERTE SÚBITA}

La muerte súbita en deportistas es multicausal. La mayoría de ellas, están relacionadas con las enfermedades cardiovasculares. Como se revisó anteriormente, el concepto de muerte súbita es muy amplio y puede abarcar cualquier tipo de evento, independientemente de su etiología ${ }^{(6,14-16)}$.

Las principales causas cardiovasculares de Muerte Súbita en Deportistas (MSD) son relacionadas con la miocardiopatía hipertrófica, las anomalías congénitas de las arterias coronarias y la displasia arritmogénica del ventrículo derecho. En deportistas menores de 35 años de edad, la causa principal de muerte súbita son las afecciones cardiacas hereditarias; entre el $40 \%$ y el $50 \%$ de los fallecimientos de deportistas jóvenes de forma súbita se deben a esta causa, y la tasa de ocurrencia aumenta cuando estos sujetos realizan ejercicio. En los deportistas mayores a 35 años de edad,la principal causa de muerte súbita es por enfermedad coronaria aterosclerótica ${ }^{(6,7,15-18)}$.

Su fisiopatología se basa en la aparición de arritmias malignas ventriculares por alteración de la conducción en un músculo malformado o por una isquemia inducida por la obstrucción del tracto de salida del ventrículo izquierdo. El 50\% de esas arritmias ventriculares malignas, se relacionan con casos de fibrilación ventricular que van precedidas por una taquicardia ventricular que es responsable de la muerte súbita. Lo anterior, es similar con los perfiles epidemiológicos mundiales, los cuales muestran que la mayor 
cantidad de paros cardiorrespiratorios se deben a arritmias ventriculares ${ }^{(14,18-}$ 22).

Existe además, una variante que es la hipertrofia idiopática del ventrículo izquierdo, que puede ser resultado de una enfermedad no diagnosticada, como la hipertensión, aunque este tipo de hipertrofia no predispone en sí misma a las arritmias durante el ejercicio ${ }^{(18,23)}$.

La segunda causa de muerte súbita es la anomalía congénita de las arterias coronarias (6). Entre las cuales se destacan múltiples malformaciones que van desde implantaciones anormales, acortamiento de vasos sanguíneos, hipoplasia del ventrículo derecho con disminución de la contractilidad, hasta displasia arritmogénica del ventrículo derecho que es una miocardiopatía idiopática, que afecta principalmente al ventrículo derecho y que provoca arritmias de manera frecuente durante el ejercicio. Esta cardiopatía silente requiere una fuerte provocación que desencadene la aparición de las arritmias malignas ${ }^{(18,21)}$.

Existen varios estudios que han demostrado que más de la mitad de estas muertes repentinas son de origen natural idiopático o de origen indeterminado y se diagnostican como muerte subida indeterminada. Algunos estudios también han demostrado casos con la alteración molecular, relacionado con los canales de los electrólitos que determinan el impulso cardíaco ${ }^{(14,24)}$.

En las últimas décadas se ha producido un enorme avance en identificación y estudios genéticos de enfermedades asociadas a muerte súbita. Se ha demostrado que en la mayoría de los casos existe mutación patogénica como responsable de la muerte. De igual forma, se ha analizado alteraciones genéticas que se producen en genes que codifican principalmente los canales de sodio, potasio y calcio, encargados de la generación eléctrica del corazón $(6,20,21)$.

Otros estudios han señalado que impactos en la región torácica, justo por encima del ventrículo izquierdo, que conlleven a contusiones cardiacas sobre todo en deportes cuerpo a cuerpo como en el karate, boxeo entre otros, se relacionan con arritmias cardiacas. Lo anterior, sobre todo si se produce en una zona vulnerable del ciclo cardíaco como es durante la repolarización ventricular, lo que rompe la estabilidad eléctrica del corazón, precipitando un latido prematuro (ectopias) que a su vez provoca una fibrilación ventricular ${ }^{(25)}$.

Sin tener en cuenta la edad y otros factores, la muerte súbita durante el ejercicio se produce casi siempre sobre una cardiopatía subyacente que origina una desestabilización eléctrica, dando lugar a una arritmia grave durante o inmediatamente después del ejercicio ${ }^{(15)}$. Es así, como se puede concluir que el deporte por sí solo, no genera la muerte súbita, requiere de factores de riesgo que predisponen el corazón.

Como factores coayudantes a la aparición de casos de muerte súbita está el consumo de sustancias como la cocaína, esteroides anabolizantes y otros agentes dopantes. El uso de estas sustancias, clasificadas como doping positivo, 
ha dado origen a una profunda preocupación por la aparición de efectos secundarios graves ${ }^{(26)}$. Experimentaciones con animales ha demostrado que el consumo de anabolizantes está muy relacionado con la muerte súbita. Es posible que el consumo de anabolizantes no sea la causa directa, sino que acelere el desarrollo de una cardiopatía subyacente y, como consecuencia de ello, la aparición de arritmias malignas ${ }^{(27)}$.

\section{PREVENCIÓN Y MANEJO INICIAL}

La prevención de la muerte súbita es una tarea difícil. Lo síntomas puede ser la primera manifestación de la enfermedad, entre ellos palpitaciones, presíncope y sincope previo al colapsó. Muchos de los deportistas han manifestado en alguna ocasión estos síntomas, por tal motivo es difícil el reconocimiento y dificulta la atención ${ }^{(15)}$.

Los equipos deportivos deben establecer procesos de valoración médica de ingreso y de manera periódica de los deportistas. El reconocimiento cardiológico previo, es punto clave para poder identificar factores de riesgo o patologías previas que puedan desencadenar arritmias fatales. Es fundamental realizar un diagnóstico diferencial entre las adaptaciones propias del deportista así denominado "corazón de atleta" y la enfermedad cardíaca $(16,21,28)$. Lo anterior, incluye aquellas personas que practican alguna actividad deportiva sin fines competitivos ${ }^{(29,30)}$.

Las evaluaciones médicas cuentan con un consenso por profesionales que han establecido unos mínimos de reconocimiento. Esta valoración está compuesta por: la historia clínica personal y familiar del deportista, la evaluación clínica y el electrocardiograma (ECG). La valoración es importante que sea realizada por personal médico especializado en el área de actividad física y el deporte. De esta manera se puede ir sumando criterios que orienten si el deportista debe ser evaluado de forma más profunda, como la necesidad de realizar pruebas de esfuerzos, ecocardiogramas, entre otros exámenes, para determinar si presenta alto riesgo cardiovascular ${ }^{(6,16,28,31)}$.

En estudios realizados en Estados Unidos y Europa se identificó que gran porcentaje de los colapsos súbitos de los deportistas fueron presenciados, pero sólo en el $30 \%$ de los casos se iniciaron las maniobras de reanimación cardiopulmonar básicas (RCP). Es así, como se debe hacer énfasis en la preparación frente a estas maniobras ${ }^{(6,32-35)}$.

Por cada minuto que un paciente no reciba $\mathrm{RCP}$ básica disminuye su probabilidad de supervivencia en un 10\%. Es así, como se hace necesario el reconocimiento precoz de víctimas en paro cardiorrespiratorio secundario a colapso súbito; además de la activación de las líneas de emergencias e iniciar maniobras de RCP con énfasis en las compresiones torácicas en el menor tiempo posible ${ }^{(5,33,36)}$.

Algunos estudios comprobaron que haber iniciado maniobras de RCP y realizar desfibrilación precoz, fueron los más fuertes predictores independientes de supervivencia en el hospital. Por lo tanto, la estrategia de prevención de la 
MSD debería incluir, el entrenamiento entre la población deportiva de maniobras básicas de RCP y la instalación de desfibriladores semiautomáticos en todos los lugares donde se concentran las actividades deportivas ${ }^{(6,32,33,37)}$. El tiempo es crítico para la supervivencia de un paciente el cual presenta un colapso subido secundario a una cardiomiopatía. Durante este tiempo, la supervivencia de la víctima depende del inicio precoz de las técnicas de reanimación cardiopulmonar.

La técnica de RCP se caracteriza por brindar ciclos de compresiones sobre el tórax del paciente, ventilaciones y el uso de un desfibrilador automático externo (DEA). Según los protocolos internacionales y consenso de investigadores, las compresiones son el elemento más importante durante la $\mathrm{RCP}$, se deben proporcionar con una profundidad de $5 \mathrm{~cm}$ no más de $6 \mathrm{~cm}$, con una frecuencia de 100 a 120 compresiones por minuto ${ }^{(36,38,39)}$. Las ventilaciones se deben brindar con dispositivos de barrera (válvulas unidireccionales), para lo cual, solo se cuenta con diez segundos para brindar dos ventilaciones entre ciclos de 30 compresiones; una pausa prolongada de las compresiones disminuye la presión de perfusión coronaria y cerebral ${ }^{(40,41)}$.

El uso del desfibrilador externo automático, se consolida como una medida salvadora, su uso requiere de entrenamiento y práctica de habilidades. Este dispositivo es la medida eficaz para la atención de casos de paro cardiorrespiratorio con ritmos de fibrilación ventricular y taquicardia ventricular sin pulso ${ }^{(33,42-49)}$.

Finalmente, las víctimas de MSD requieren maniobras de reanimación cardiopulmonar precoz, lo que proporciona flujo sanguíneo al cerebro y corazón, mientras se le provee una descarga eléctrica con un desfibrilador que es el tratamiento necesario para revertir los ritmos de paro presentes o mientras se soporta al paciente mientras acude personal más experimentado, entrenado y aborden el manejo definitivo de este $(16,28,33,50)$.

\section{CONCLUSIONES}

- La muerte súbita en deportistas cuenta con varios estudios recientes, con artículos científicos destacados en los últimos dos años (2016 - 2017). Las investigaciones realizadas se destacan en diferentes lugares y cuentan con un análisis detallado.

- Los artículos revisados muestran que la principal causa de mortalidad en los deportistas son cardiopatías congénitas y la enfermedad ateroesclerótica.

- Los deportistas de alto rendimiento requieren exámenes previos y periódicos por parte de recurso humano calificado, de tal forma, que se identifique de manera temprana factores de riesgo de muerte súbita.

- Es determinante que los equipos deportivos, entrenadores, deportistas y en general todos los primeros respondientes a una muerte súbita, cuenten con entrenamiento de habilidades y destrezas de reanimación cardiopulmonar. 
- La instalación, capacitación y el uso de los desfibriladores externos automáticos, se constituyen en elementos esenciales para disminuir la mortalidad de los atletas en escenarios deportivos.

\section{REFERENCIAS}

1. Narayanan K, Bougouin W, Sharifzadehgan A, Waldmann V, Karam N, Marijon E, et al. Sudden Cardiac Death During Sports Activities in the General Population. Cardiac Electrophysiology Clinics. 2017 Dec 1;9(4):559-67.

2. DeCS - Descriptores en Ciencias de la Salud [Internet]. [cited 2017 Dec 6]. Available from: $h$ ttp://decs.bvs.br/E/homepagee.htm

3. Katch RK, Scarneo SE, Adams WM, Armstrong LE, Belval LN, Stamm JM, et al. Top 10 Research Questions Related to Preventing Sudden Death in Sport and Physical Activity. Res Q Exerc Sport. 2017 Sep;88(3):251-68.

4. Gutiérrez Sotelo O, Gutiérrez Sotelo O. Muerte súbita en deportistas. Revista Costarricense de Cardiología. 2014 Dec;16(2):18-24.

5. Asif IM, Harmon KG. Incidence and Etiology of Sudden Cardiac Death: New Updates for Athletic Departments. Sports Health. 2017 Jun;9(3):268-79.

6. Corrado D, Zorzi A. Sudden death in athletes. Int J Cardiol. 2017 Jun 15;237:6770 .

7. Wasfy MM, Hutter AM, Weiner RB. Sudden Cardiac Death in Athletes. Methodist Debakey Cardiovasc J. 2016;12(2):76-80.

8. Vandenberg JI, Perry MD, Hill AP. Recent advances in understanding and prevention of sudden cardiac death. F1000Res [Internet]. 2017 Aug 31 [cited 2017 Dec 12];6. Available from: https://www.ncbi.nlm.nih.gov/pmc/articles/PMC5583740/

9. di Gioia CRT, Giordano C, Cerbelli B, Pisano A, Perli E, De Dominicis E, et al. Nonischemic left ventricular scar and cardiac sudden death in the young. Human Pathology. 2016 Dec 1;58(Supplement C):78-89.

10. García-Ortiz JD, García-Ortiz JD. Heart screening for prevention of sudden death in college athletes from Universidad de Antioquia, Medellin, Colombia. latreia. 2017 Sep;30(3):265-75.

11. Lang CN, Steinfurt J, Odening KE. Avoiding sports-related sudden cardiac death in children with congenital channelopathy: Recommendations for sports activities. Herz. 2017 Apr;42(2):162-70.

12. Mascia G, Arbelo E, Ojeda JH, Solimene F, Brugada R, Brugada J. Brugada Syndrome and Exercise Practice: Current Knowledge, Shortcomings and Open Questions. Int J Sports Med. 2017 Jul;38(8):573-81.

13. Martens E, Sinner MF, Siebermair J, Raufhake C, Beckmann BM, Veith S, et al. Incidence of sudden cardiac death in Germany: results from an emergency medical service registry in Lower Saxony. Europace. 2014 Dec;16(12):1752-8. 
14. Vargas Sanabria M, Chaves V, Carlos J, Hernández Romero G, Montero Solano G, Vargas Sanabria M, et al. Síndrome de brugada como casusa de muerte súbita de origen cardíaco. Medicina Legal de Costa Rica. 2017 Mar;34(1):157-64.

15. Sitges M, Brugada J. Sudden death in the athlete. Med Clin (Barc). :540-2.

16. Morin DP, Homoud MK, Estes NAM. Prediction and Prevention of Sudden Cardiac Death. Cardiac Electrophysiology Clinics. 2017 Dec 1;9(4):631-8.

17. Álvarez-Ortiz A, Gutiérrez SN, Martínez E, León MD, Mariño-Murillo L, MoraBautista VM, et al. Miocardiopatía arritmogénica del ventrículo derecho. revmed. 2015 Jun 22;27(3):123-34.

18. Tang PT, Shenasa M, Boyle NG. Ventricular Arrhythmias and Sudden Cardiac Death. Cardiac Electrophysiology Clinics. 2017 Dec 1;9(4):693-708.

19. Dorantes M, Marrero R, Méndez A, Castro J, Vázquez A. Historia familiar de muerte súbita por fibrilación ventricular idiopática. Archivos de cardiología de México. 2014 Mar;84(1):57-9.

20. Campuzano O, Sanchez-Molero O, Fernandez A, Iglesias A, Brugada R. Muerte súbita cardiaca de origen arrítmico: valor del análisis genético post mortem. Rev Esp Med Legal [Internet]. [cited 2017 Dec 7]; Available from: http://www.elsevier.es/es-revista-revista-espanola-medicina-legal-285-avanceresumen-muerte-subita-cardiaca-origen-arritmico-S0377473217300706

21. Semsarian C, Ingles J. Preventing sudden cardiac death in athletes. BMJ. 2016 Apr 20;353:i1270.

22. American Heart Association. Soporte Vital Cardiovascular Avanzado. 2016.

23. Tereshchenko LG, Soliman EZ, Davis BR, Oparil S. Risk stratification of sudden cardiac death in hypertension. J Electrocardiol. 2017 Dec;50(6):798-801.

24. Araya Vargas J, Araya Vargas J. Canalopatías en muerte súbita: Relevancia clínica de autopsia molecular. Medicina Legal de Costa Rica. 2017 Mar;34(1):272-8.

25. Alvarado C, Vargas F, Guzmán F, Zárate A, Correa JL, Ramírez A, et al. Trauma cardiaco cerrado. Revista Colombiana de Cardiología. 2016 Jan 1;23(1):49-58.

26. Morentin B, Callado LF, García-Hernández S, Bodegas A, Lucena J. Papel de las sustancias tóxicas en la muerte súbita cardiaca. Revista Española de Medicina Legal [Internet]. 2017 Jul 29 [cited 2017 Dec 7]; Available from: http://www.sciencedirect.com/science/article/pii/S0377473217300469

27. Avella RE, Medellín JP. Los esteroides anabolizantes androgénicos, riesgos y consecuencias. Revista UDCA Actualidad \&amp; Divulgación Científica. 2012 May;15:47-55.

28. Chappex N, Schlaepfer J, Fellmann F, Bhuiyan ZA, Wilhelm M, Michaud K. Sudden cardiac death among general population and sport related population in forensic experience. J Forensic Leg Med. 2015 Oct;35:62-8.

29. Schmied CM. Improvement of cardiac screening in amateur athletes. $J$ Electrocardiol. 2015 Jun;48(3):351-5. 
30. Asperti AM, Fernandes TL, Pedrinelli A, Hernandez AJ. Sports injuries among amateur athletes at a brazilian university. Acta Ortop Bras. 2017;25(2):93-8.

31. Borrione P, Quaranta F, Ciminelli E. Pre-participation screening for the prevention of sudden cardiac death in athletes. World J Methodol. 2013 Mar 26;3(1):1-6.

32. García Reyes J. Reanimación cerebrocardiopulmonar prolongada exitosa en un paciente con muerte súbita: un reporte de caso. Rev Colomb Anestesiol. 2014 Jul $1 ; 42(3): 229-33$.

33. Kwon Y, Aufderheide TP. Optimizing Community Resources to Address Sudden Cardiac Death. Cardiac Electrophysiology Clinics. 2009 Dec 1;1(1):41-50.

34. Brown LE, Lynes C, Carroll T, Halperin H. CPR Instruction in U.S. High Schools: What Is the State in the Nation? J Am Coll Cardiol. 2017 Nov 28;70(21):2688-95.

35. Onan A, Simsek N, Elcin M, Turan S, Erbil B, Deniz KZ. A review of simulationenhanced, team-based cardiopulmonary resuscitation training for undergraduate students. Nurse Educ Pract. 2017 Nov;27:134-43.

36. American Heart Association. SVB / BLS para profesionales de la salud. 2016.

37. García Reyes J. Reanimación cerebrocardiopulmonar prolongada exitosa en un paciente con muerte súbita: un reporte de caso. Revista Colombiana de Anestesiología. 2014 Jul 1;42(3):229-33.

38. Ramli R, Oxley J, Hillard P, Mohd Sadullah AF, McClure R. The effect of motorcycle helmet type, components and fixation status on facial injury in Klang Valley, Malaysia: a case control study. BMC Emergency Medicine. 2014 Aug 3;14:17.

39. Nassar BS, Kerber R. Improving CPR Performance. Chest. 2017 Nov;152(5):10619.

40. Ackerman M, Atkins DL, Triedman JK. Sudden cardiac death in the young. Circulation. 2016 Mar 8;133(10):1006-26.

41. Meaney PA, Bobrow BJ, Mancini ME, Christenson J, de Caen AR, Bhanji F, et al. Cardiopulmonary resuscitation quality: [corrected] improving cardiac resuscitation outcomes both inside and outside the hospital: a consensus statement from the American Heart Association. Circulation. 2013 Jul 23;128(4):417-35.

42. Leal-Forero LC, Martínez-Malo LC, Navarro-Vargas JR. Brain cardiopulmonary reanimation: state of the art. Revista de la Facultad de Medicina. 2014 Jan;62(1):149-55.

43. Gempeler R. FE. Reanimación cardiopulmonar. Más allá de la técnica. Revista Colombiana de Anestesiología. 2015 Apr 1;43(2):142-6.

44. Aranzábal-Alegría G, Verastegui-Díaz A, Quiñones-Laveriano DM, QuintanaMendoza LY, Vilchez-Cornejo J, Espejo CB, et al. Factors influencing the level of knowledge of cardiopulmonary resuscitation in hospitals in Peru. Colombian Journal of Anesthesiology. 2017 Apr 1;45(2):114-21. 
45. Navarro-Vargas JR, Matiz-Camacho H, Osorio-Esquivel J. Manual de práctica clínica basado en la evidencia: Reanimación cardiocerebropulmonar. Rev Colomb Anestesiol. 2015 Jan 1;43(1):9-19.

46. Mitani $\mathrm{Y}$, Ohta K, Ichida F, Nii M, Arakaki Y, Ushinohama H, et al. Circumstances and Outcomes of Out-Of-Hospital Cardiac Arrest in Elementary and Middle School Students in the Era of Public-Access Defibrillation. Circulation Journal. 2014;78(3):701-7.

47. Niggemeyer Á, Xalambrí $F$, Albornoz H, Sánchez A, López $P$, Urroz N, et al. Resucitación utilizando desfibrilador externo automático en el Uruguay. Revista Uruguaya de Cardiología. 2015 Apr;30(1):32-8.

48. Orjuela Guerrero A. Cardioversión eléctrica en fibrilación auricular. Rev Colomb Cardiol. :57-64.

49. Nichol G, Sayre MR, Guerra F, Poole J. Defibrillation for Ventricular Fibrillation: A Shocking Update. J Am Coll Cardiol. 2017 Sep 19;70(12):1496-509.

50. Kurata $H$, Ishigami A, Tokunaga I, Nagasaki $Y$, Nishimura A. Sudden cardiac death during first-time jogging. J Med Invest. 2017;64(1.2):184-6.

Referencias totales citadas: 50

Referencias citadas correspondientes a la Rev lb CC Act Fis Dep: 0 